\title{
Crescimento de Espécies de Brachiaria sob Déficit Hídrico e Alagamento a Campo'
}

\author{
Jorge Luiz Schirmer de Mattos², José Alberto Gomide ${ }^{3}$, Carlos Alberto Martinez y Huaman ${ }^{4}$
}

RESUMO - Dois experimentos foram conduzidos, sendo um instalado em área de encosta e outro em área de baixada. Objetivou-se avaliar a tolerância à seca e ao alagamento de três espécies comerciais de Brachiaria (B. decumbens cv. Basilisk, B. brizantha cv. Marandu e B. mutica) e três acessos (B. brizantha B-11, B. humidicola cv. Tupi e B. dictyoneura), a partir de suas características morfogênicas, produtivas e relações hídricas. O delineamento experimental foi o de blocos ao acaso, com seis repetições. As parcelas, com $4 \mathrm{~m}^{2}$ de área total, foram constituídas de oito linhas de $2 \mathrm{~m}$ de comprimento, com espaçamento entre linhas de $0,25 \mathrm{~m}$. Dezenove dias após corte de uniformização, a área localizada na baixada foi submetida ao alagamento de uma lámina d'àgua de $56 \mathrm{~mm}$ de água, por 30 dias; simultaneamente, suspendeu-se a irrigação na área de encosta sob déficit hídrico. O alagamento foi mais prejudicial às características morfogênicas que déficit hídrico. Enquanto a taxa de alongamento foliar de todas as espécies foi mais baixa sob alagamento, as taxas de aparecimento e senescência foliares responderam ao estresse hídrico conforme a espécie. A taxa de aparecimento de folhas foi mais comprometida pelo alagamento apenas em B. dictyoneura, B. mutica e B. brizantha cv. Marandu. As maiores taxas de senecência de folhas ocorreram sob alagamento, particularmente em B. decumbens, B. brizantha cv. Marandu e B-11. Os estresses hídricos tiveram efeitos diferenciados sobre as características produtivas das espécies. A produção de biomassa verde aérea foi maior em $B$ - 11 sob regime de déficit hídrico, enquanto em B. dictyoneura e B. mutica, observou-se tendência de valores mais altos sob regime de alagamento. $\mathrm{O}$ índice de área foliar foi mais alto sob deficit hídrico, apenas em B. brizantha Marandu e B-11. A população de perfilhos basilares foi maior sob alagamento apenas em B. dictyoneura.

Palavras-chave: biomassa, status foliar hídrico, índices morfogênicos, perfilhamento

\section{Effect of Water Deficit and Flooding on the Growth of Brachiaria Species in the Field}

ABSTRACT - Two experiments were carried out from December/1997 to June/1998. One experiment was performed on hilly area e and the second one on lowland area. These experiments aimed to evaluate the tolerance to flooding and low soil water potential of three commercial Brachiaria species (B. decumbens cv. Basilisk, B. brizantha cv. Marandu e B. mutica) and three acesses (B. brizantha B-11, B. humidicola $\mathrm{cv}$. Tupi and B. dictyoneura). Morphogenetical, productive and plant water status were the variables assessed. In each experiment the species were distributed in randomized blocks with six replications. Each plot had eight $2 \mathrm{~m}$ rows, spaced $0.25 \mathrm{~m}$ from each other. During the establishment phase both experimental areas were irrigated, if required. Nineteen days after uniformization clipping, the experiment on the lowland was daily flooded with a $56 \mathrm{~mm}$ of water lamina over 30 days, while the irrigation was supressed on the hilly area. Flooding was more detrimental to the morphogenetic traits of the Brachiaria species than water deficit. All species exhibited lower rate of leaf elongation under flooding but their leaf appearance and senescence rate responses to flooding varied with the species. Rate of leaf blade appearance in B. dictioneura, B. mutica and B. brizantha cv. Marundu was higher under water deficit than under flooding; leaf blade senecence rate was higher under flooding, particularly in B. decumbens and B. brizantha cv. Marandu and B-11. Flooding and water deficit effects on the productive traits varied with species. Green shoot biomass production was higher in B-11 under water deficit but in $B$. dictyoneura and $B$. mutica these values tended to be higher under flooding. Higher leaf area index figures were observed under water deficit only for B. brizantha Marandu and B-11. Under flooding basal tiller population was higher in B. dictyoneura. B. humidicola showed little variation with stress situation for most studied traits, while B. brizantha B-11 performed better under water deficit than under flooding.

Key Words: leaf water status, morphogenetical indices, plant biomass, tillering

\section{Introdução}

Fatores associados às taxas de aparecimento, de alongamento e de senescência das lâminas foliares podem afetar a produção de forragem, condicionando a produção animal.
As taxas de aparecimento e de alongamento e o tempo de vida das folhas, principais constituintes das características morfogênicas do perfilho, são determinantes do número e do tamanho das folhas e da densidade de perfilhos - denominados características estruturais do dossel e responsáveis pelo índice

\footnotetext{
${ }^{1}$ Parte da tese do primeiro autor.

2 Estudante de doutorado, Departamento Zootecnia, UFV, bolsista do CNPq.

3 Bolsista do CNPq, Departamento de Zootecnia, UFV (jagomide@ufv.br).

${ }^{4}$ Professor de Fisiologia Vegetal da USP, Ribeirão Preto- SP(carlosamh@ffclrp.usp.br).
} 
de área foliar da pastagem - e podem ser condicionados por fatores abióticos, como água, luz, temperatura e nutrientes (Chapman \& Lemaire, 1993).

Áreas de pastagens sujeitas a veranicos ou secas estacionais e alagamento são comuns em quase todo o território nacional. As espécies do gênero Brachiaria são as mais usadas no estabelecimeto, na formação e recuperação de pastagens no Brasil (Zimmer \& Euclides, 2000). Todavia, poucos são os estudos envolvendo as características que conferem adaptação à seca ou ao alagamento a estas espécies.

B. decumbens Stapf e B. brizantha (Hochst ex A.Rich) Stapf cv Marandu são consideradas tolerantes à seca, enquanto a B. humidicola (Rendle) Schweickerdt, a B. dictyoneura (Fig.\& De Not.) Stapf e a $B$. mutica (Forsk) Stapf são tolerantes ao alagamento (Soares Filho, 1994). Relações entre o hábito de crescimento e a morfologia com tolerância à seca e ao alagamento têm sido observadas. Espécies de hábito cespitoso são mais tolerantes à seca que espécies estoloníferas (Jones et al., 1980; Bittmann \& Simpson, 1987). Espécies estoloníferas e de porte alto, por sua vez são mais tolerantes ao alagamento (Anderson, 1974). Entretanto, Etherington (1984) sugere que plantas de porte baixo são mais tolerantes ao alagamento que as de porte alto, enquanto Baruch (1994 a,b) afirma que espécies estoloníferas são mais tolerantes ao alagamento que à seca. Folhas estreitas e pequenas conferem resistência à seca (Klar et al., 1978; Bittman \& Simpson, 1987) e ao alagamento (Etherington, 1984).

Alcântara (1987), Soares Filho (1994) e Valle et al. (2000), revisando sobre a morfologia de espécies de Brachiaria, observaram que a $B$. decumbens cv. Basilisk caracteriza-se por apresentar plantas suberetas ou prostradas, pequenos rizomas, poucas raízes adventícias; a $B$. brizantha cv. Marandu é cespitosa, com rizomas muito curtos e poucas raízes adventícias; a B. humidicola possui hábito prostrado, é fortemente estolonífera, com rizomas em nódulos curtos ou longos e finos e raízes adventícias nos estolões; $B$. dictyoneura apresenta hábito cespitoso e ausência de estolões; e B. mutica apresenta colmos prostrados, rizomas curtos e vigorosos e raízes adventícias numerosas.

Objetivou-se avaliar, sob condições de campo, a tolerância à seca e ao alagamento de espécies de Brachiaria, sendo três cultivares (B. decumbens cv. Basilisk, B. brizantha cv. Marandu, B. mutica) e três acessos (B. brizantha B-11, B. humidicola cv. Tupi e B. dictyoneura).

\section{Material e Métodos}

Dois experimentos foram conduzidos, no período de dezembro de 1997 a junho de 1998, na Universidade Federal de Viçosa, em Viçosa, MG, Brasil (latitude $20^{\circ} 45^{\prime} \mathrm{S}$, longitude $42^{\circ} 51^{\prime} \mathrm{W}$, altitude $650 \mathrm{~m}$ ): um instalado em área de encosta (20\%) e outro em área de baixada. Em ambos, foram avaliadas três espécies comerciais do gênero Brachiaria (B.decumbens cv. Basilisk, B. brizantha cv. Marandu e B. mutica) e três acessos (B. brizantha B-11, B. humidicola cv. Tupi e $B$. dictyoneura). O delineamento experimental de cada experimento foi o de blocos ao acaso, com seis repetições.

Amostras de solo da área experimental, relativas à camada de 0-20 cm de profundidade, revelaram composição granulométrica de 0,35 e $0,16 \mathrm{~kg} / \mathrm{kg}$ de areia grossa, 0,16 e $0,8 \mathrm{~kg} / \mathrm{kg}$ de areia fina, 0,5 e $0,22 \mathrm{~kg} / \mathrm{kg}$ de silte e 0,44 e $0,54 \mathrm{~kg} / \mathrm{kg}$ de argila, além da seguinte composição química: 5,0 e $6,5 \mathrm{mg} / \mathrm{dm}^{3}$ de P; 96 e $29 \mathrm{mg} / \mathrm{dm}^{3}$ de $\mathrm{K} ; 1,7$ e 3,9 $\mathrm{cmol}_{\mathrm{c}}$ de $\mathrm{Ca}^{2+}$ e 0,6 e $0,7 \mathrm{cmol}_{\mathrm{c}} / \mathrm{dm}^{3}$ de $\mathrm{Mg}^{2+}$ e $\mathrm{pH}$ em água de 5,4 e 5,9, respectivamente, nas áreas de encosta e de baixada. Foi revelada também ausência de alumínio.

O solo recebeu preparo convencional de aração, seguida de gradeação. Foi efetuada calagem, 1.330 e $930 \mathrm{~kg} / \mathrm{ha}$ de calcário dolomítico, nas áreas de encosta e de baixada, respectivamente, e incorporação do calcário ao solo com grade niveladora, 30 dias antes da semeadura/plantio.

A adubação por ocasião da semeadura, realizada em 24/12/1997, consistiu na aplicação de 555,100 e $555 \mathrm{~kg} / \mathrm{ha}$ de superfosfato simples, cloreto de potássio e sulfato de amônio, respectivamente. Em 02/05/1998, realizou-se a adubação em cobertura com 33 e $277 \mathrm{~kg} / \mathrm{ha}$ de cloreto de potássio e sulfato de amônio, respectivamente, nas duas áreas.

As parcelas, com área total de $4 \mathrm{~m}^{2}$, eram constituídas de oito linhas de $2 \mathrm{~m}$ de comprimento, com espaçamento de $0,25 \mathrm{~m}$, e área útil de $1 \mathrm{~m}^{2}$ por parcela, correspondente às quatro linhas centrais, após eliminação de $0,50 \mathrm{~m}$ de bordadura, nas extremidades.

As espécies foram estabelecidas via sementes, fornecidas pelo Centro Nacional de Pesquisa de Gado de Corte, da Empresa Brasileira de Pesquisa Agropecuária, exceto a B. mutica, que foi estabelecida por meio de propagação vegetativa. 
O período de estabelecimento das espécies estendeu-se até 30/04/1998, quando se praticou o corte de uniformização, a $10 \mathrm{~cm}$ acima do solo.

Durante o período de estabelecimento, a precipitação pluvial $(490 \mathrm{~mm})$ foi complementada com $89 \mathrm{~mm}$ de água, via irrigação por aspersão, aplicada estrategicamente nos momentos considerados mais críticos de disponibilidade de água no solo, de modo a se obter sucesso no estabelecimento. A precipitação pluvial de $38 \mathrm{~mm}$ ocorrida no período entre o corte de uniformização e a imposição das situações de estresse foi complementada com aproximadamente $26 \mathrm{~mm}$, via água de irrigação por aspersão.

A partir de 19/05/1998, os estresses hídricos foram provocados pela suspensão da irrigação na àrea de encosta e pelo alagamento na área de baixada. $\mathrm{O}$ experimento da baixada foi inundado, diariamente, por uma lámina dàgua de aproximadamente $56 \mathrm{~mm}$ (28 mm de manhã e à tarde). Também a partir desta data, suspendeu-se a irrigação no experimento sob décit hídrico. Entretanto, registrou-se precipitação de 34 mm durante o estresse (19/05 a 18/06).

A temperatura e a umidade relativa do ar, registrados em uma mini-estação meteorológica instalada próximo à área experimental, alcançaram valores médios de $17,3 \pm 2,1^{\circ} \mathrm{C}$ e $81,1 \pm 5,84 \%$, respectivamente, após o corte de uniformização.

Em 18/06, a fim de se estimar a disponibilidade de água no solo do experimento sob déficit hídrico, coletaram-se amostras de solo, uma por parcela, a 0-40 cm de profundidade, utilizando-se um trado tipo caneca com $5 \mathrm{~cm}$ de diâmetro. Cada amostra de solo foi acondicionada em uma cápsula de alumínio, pesada e, posteriormente, levada a estufa a $105^{\circ} \mathrm{C}$, para a determinação do teor de água no solo. A partir destes valores, estimou-se o potencial hídrico do solo, de acordo com a curva característica de retenção de água no solo ( $\hat{\mathrm{Y}}=0,159425 . \Psi$ solo $\left.^{-0,09995} ; \mathrm{R}^{2}=0,94\right)$, obtendo-se valor médio de 1,09 +/- 0,02 MPa.

As taxas de aparecimento, alongamento e senescência de lâminas foliares foram avaliadas segundo Davies (1993), durante 28 dias (19/05 a 16/06/ 1998). O potencial hídrico de lâminas foliares $\left(Y_{1}\right)$ foi determinado em 20/05, 03/06, 07/06, 11/06 e 14/06/ 1998. As medições do $Y_{1}$ foram feitas, sempre no mesmo horário solar, de 7 a $8 \mathrm{~h}$, utilizando-se as lâminas foliares recém-expandidas.

Em 18/06/1998, realizou-se corte rente ao solo, colhendo-se a biomassa da parte aérea presente na área útil das parcelas de ambos os experimentos. $\mathrm{O}$ material vegetal colhido foi pesado em balança tipo dinamômetro com divisões de $100 \mathrm{~g}$, a fim de se conhecer a produção de forragem verde por área. Antes disso, colheu-se uma amostra de forragem, cortada rente ao solo, e do material morto sobre o solo, em uma área de $0,0625 \mathrm{~m}^{2}$, delimitada por um quadrado de 25 x $25 \mathrm{~cm}$. O material vegetal, cujo peso foi adicionado ao peso total da área útil, foi separado em parte aérea verde (lâminas e colmos + bainhas) e parte aérea morta (partes de lâminas, colmos e bainhas destacadas das plantas durante o processo de separação; acrescidas da "liteira"), a fim de se determinar o peso da matéria fresca destas porções. Uma amostra representativa das lâminas foliares foi destinada à medição da área de lâminas foliares.

Amostras representativas das porções referentes à parte aérea permaneceram por três a sete dias em estufa de ventilação forçada a $70^{\circ} \mathrm{C}$ até atingirem peso constante, com vistas à obtenção do peso da matéria seca.

As características da biomassa, avaliadas nos experimentos de déficit hídrico e de alagamento, foram índice de área foliar, matéria verde seca total e de lâminas foliares, matéria morta seca da parte aérea, matéria seca do sistema radicular, número de perfilhos basilares e aéreos.

Para a recuperação do sistema radicular, foram feitas duas amostragens por parcela, apenas no experimento sob déficit hídrico, a $0-40 \mathrm{~cm}$ de profundidade, com auxílio de um trado tipo caneca, de $5 \mathrm{~cm}$ de diâmetro. As duas amostras de cada parcela deram origem a uma amostra composta, que foi seca em estufa a $70^{\circ} \mathrm{C}$, para a determinação do peso da matéria seca do sistema radicular. De posse do peso da matéria seca do sistema radicular, com base no respectivo volume de solo colhido por parcela, procedeu-se aos cálculos, para a estimativa da produção de matéria seca do sistema radicular por hectare.

Também se calculou a relação entre os dados de produção de biomassa total da parte aérea (matérias seca verde e morta) registrados durante os períodos de déficit hídrico e de alagamento e seus respectivos valores, em termos percentuais, na fase de estabelecimento, em que as condições de cultivo foram favoráveis ao crescimento das plantas, com o propósito de se determinar a tolerância à seca e ao alagamento das espécies de Brachiaria.

Informações complementares concernentes aos 
métodos e equipamentos utilizados no presente experimento encontram-se em Mattos (2001).

Para as análises estatísticas, consideraram-se os tratamentos arranjados em esquema fatorial $6 \times 2$ (seis espécies e duas situações de estresse - déficit hídrico e alagamento), em um delineamento experimental de blocos ao acaso, com seis repetições.

Os dados experimentais foram submetidos à análise de variância conjunta (Banzatto \& Kronka, 1992), que teve como fontes de variação espécie, situação de estresse, espécie x situação de estresse, blocos dentro de situação de estresse e erro experimental. Exceção foi feita ao potencial hídrico de lâminas foliares avaliado em 11/06, que foi submetido à análise de variância, em cada experimento, segundo um delineamento de blocos ao acaso, com seis repetições, compreendendo as seis espécies e totalizando 36 unidades experimentais. Neste caso, as fontes de variação na análise de variância foram: bloco, espécie e erro experimental. Este procedimento ocorreu em virtude de a ordem de grandeza dos quadrados médios residuais das análises individuiais dos experimentos exceder a relação de 7:1 (Banzatto \& Kronka, 1992). Dos dados de biomassa do sistema radicular, somente foram analisados aqueles referentes ao experimento de déficit hídrico. As médias dos tratamentos foram comparadas pelo teste Tukey, a 5\% de probabilidade. Para facilitar o procedimento das análises estatísti- cas, os valores do potencial hídrico de lâminas foliares foram analisados como valores absolutos. Os dados do potencial hídrico do solo do experimento sob déficit hídrico são apresentados como valores médios \pm desvio-padrão.

\section{Resultados e Discussão}

Independentemente da natureza do estresse (déficit hídrico ou alagamento), as taxas de aparecimento de lâminas foliares em B. mutica, B. humidicola e $B$. dictyoneura se igualaram e superaram $(\mathrm{P}<0,05)$ as de $B$. decumbens e B. brizantha cv. Marandu e acesso B-11, que também não diferiram entre si $(\mathrm{P}>0,05)$ sob nenhuma das situações de estresse (Tabela 1). A taxa de aparecimento de lâminas foliares foi mais alta sob a situação de déficit hídrico que de alagamento em $B$. brizantha cv. Marandu, $B$. dictyoneura e $B$. mutica, mas não variou em função da natureza do estresse nas outras espécies.

As mais altas taxas $(\mathrm{P}<0,05)$ de alongamento de lâminas foliares foram observadas em $B$. mutica, independentemente da natureza do estresse hídrico (Tabela 1). Todas as espécies apresentaram menores taxas de alongamento de lâminas foliares sob alagamento. Estas menores taxas de crescimento sob alagamento são atribuíveis à menor concentração de nutrientes, principalmente nitrogênio, nas lâminas

Tabela 1 - Taxas de aparecimento, alongamento e senescência de lâminas foliares de espécies de Brachiaria, em função da condição de estresse hídrico

Table 1 - Leaf blade appearance, elongation and senescence rates of the Brachiaria, as a function of soil moisture condition stress

\begin{tabular}{|c|c|c|c|c|c|c|}
\hline \multirow[t]{2}{*}{$\begin{array}{l}\text { Estresse }^{1} \\
\text { Stress }\end{array}$} & \multicolumn{6}{|c|}{$\begin{array}{l}\text { Espécie }^{2} \\
\text { Specie }^{2}\end{array}$} \\
\hline & $\mathrm{BD}$ & $\mathrm{BB}$ & B-11 & $\mathrm{BH}$ & BI & $\mathrm{BM}$ \\
\hline & \multicolumn{6}{|c|}{$\begin{array}{c}\text { Taxa de aparecimento (lâmina/dia.perfilho) } \\
\text { Appearance rate (leaf/day.tiller) }\end{array}$} \\
\hline \multirow[t]{2}{*}{$\mathrm{AL}$} & $\begin{array}{c}0,10 \mathrm{Ba} \\
0,08 \mathrm{Ba}\end{array}$ & $\begin{array}{c}0,10 \mathrm{Ba} \\
0,07 \mathrm{Bb}\end{array}$ & $\begin{array}{c}0,08 \mathrm{Ba} \\
0,06 \mathrm{Ba}\end{array}$ & $\begin{array}{l}0,18 \text { Aa } \\
0,16 \text { Aa }\end{array}$ & $\begin{array}{l}0,20 \mathrm{Aa} \\
0,13 \mathrm{Ab}\end{array}$ & $\begin{array}{c}0,19 \mathrm{Aa} \\
0,16 \mathrm{Ab}\end{array}$ \\
\hline & \multicolumn{6}{|c|}{$\begin{array}{c}\text { Taxa de alongamento }(\mathrm{mm} / \text { dia.perfilho }) \\
\text { Elongation rate }(\mathrm{mm} / \text { day.tiller })\end{array}$} \\
\hline \multirow[t]{2}{*}{$\mathrm{AL}$} & $\begin{array}{l}27,41 \mathrm{Ca} \\
13,07 \mathrm{Bb}\end{array}$ & $\begin{array}{l}39,98 \mathrm{Ba} \\
18,46 \mathrm{Bb}\end{array}$ & $\begin{array}{l}36,98 \mathrm{Ba} \\
17,62 \mathrm{Bb}\end{array}$ & $\begin{array}{l}12,74 \mathrm{Da} \\
5,44 \mathrm{Cb}\end{array}$ & $\begin{array}{l}14,85 \mathrm{Da} \\
5,24 \mathrm{Cb}\end{array}$ & $\begin{array}{l}56,89 \mathrm{Aa} \\
27,88 \mathrm{Ab}\end{array}$ \\
\hline & \multicolumn{6}{|c|}{$\begin{array}{c}\text { Taxa de senescência }(\mathrm{mm} / \text { dia.perfilho }) \\
\text { Senescence rate ( } \mathrm{mm} / \text { day.tiller })\end{array}$} \\
\hline $\mathrm{DH}$ & $0,17 \mathrm{Ab}$ & $0,02 \mathrm{Ab}$ & $0,0 \mathrm{Ab}$ & 0,06Aa & 0,37Aa & $0,51 \mathrm{Aa}$ \\
\hline $\mathrm{AL}$ & $4,02 \mathrm{Aa}$ & $3,29 \mathrm{ABa}$ & $2,35 \mathrm{Ba}$ & $0,32 \mathrm{Ca}$ & $0,19 \mathrm{Ca}$ & $0,64 \mathrm{Ca}$ \\
\hline
\end{tabular}

$\mathrm{BD}=$ B. decumbens; $\mathrm{BB}=B$. Brizantha Marandu; $\mathrm{B}-11=$ acesso de $B$. brizantha; $\mathrm{BH}=B$. humidicola; $\mathrm{BI}=B$. dictyoneura; $\mathrm{BM}=B$. mutica

$1 \mathrm{DH}$ : déficit hídrico (low soil moisture); $\mathrm{AL}$ : alagamento (flooding).

$\mathrm{A}>\mathrm{B}>\mathrm{C}>\mathrm{D}(\mathrm{P}<0,05)$ compara espécies (compares species); $\mathrm{a}>\mathrm{b}$ compara estresse (compares stress). 
foliares (Baruch, 1994a). De fato, segundo Watson et al. (1976), a aplicação de altas doses de nitrogênio ameniza os danos causados pelo alagamento em espécies de cereais, decorrentes da denitrificação e dos impedimentos na absorção de nutrientes.

É provável que a aplicação de $277 \mathrm{~kg} / \mathrm{ha}$ de sulfato de amônio em cobertura, logo após o corte de uniformização tenha contribuído para amenizar possível deficiência de nitrogênio nas plantas de Brachiaria sob alagamento.

A senescência de lâminas foliares variou com a situação de estresse, de modo que as maiores taxas foram registradas em solo alagado em $B$. decumbens, B. brizantha cv. Marandu e acesso B-11 (Tabela 1).

Acredita-se que a ocorrência da chuva de $34 \mathrm{~mm}$ durante o período de déficit hídrico pouco contribuiu para amenizar o efeito desse estresse sobre as espécies de Brachiaria, uma vez que a evapotranspiração em pastagens da região Sudeste, durante o período de seca, é de aproximadamente $4 \mathrm{~mm} / \mathrm{dia}$ (Weigand et al., 1998). Assim, admitindo-se este valor para a evapotranspiração observada neste estudo, isso representaria demanda evapotranspiratória de cerca de $112 \mathrm{~mm}$ para o período de 28 dias de estresse, que implicaria em "balanço hídrico" negativo de $78 \mathrm{~mm}$. Ademais, o período de 28 dias sem irrigação parece ter sido suficiente para diminuir sensivelmente a disponibilidade de água do solo, cujo potencial hídrico foi de aproximadamente -1,09 MPa na camada de 0-40 cm de profundidade no experimento sob déficit hídrico. Salienta-se, ainda, a ocorrência de acentuadas rachaduras no solo, fato que, per se, evidencia acentuada deficiência de água no solo. Por outro lado, o tratamento de alagamento não resultou em inundação permanente do solo.

$\mathrm{O}$ aparente maior efeito do alagamento sobre as taxas de alongamento foliar que de senescência foliar é atribuído às baixas temperaturas durante o período experimental, amenizando o efeito do alagamento sobre a senescência e morte de folhas (Beard \& Martin, 1970). McIvor (1984) também sugere que a senescência é mais rápida sob condições favoráveis de crescimento e que se correlaciona mais estreitamente com o suprimento de umidade que com a temperatura.

É notória a superioridade da taxa de aparecimento e alongamento de folhas de B. mutica sobre as demais espécies, tanto sob a situação de alagamento como de déficit hídrico, embora esta espécie também tenha sido sensível ao alagamento. É possível supor que o fato de a $B$. mutica não ter apresentado abundante produção de raízes adventícias tenha contribuído para a sua relativa sensibilidade ao alagamento.

Ressalta-se que a $B$. decumbens e a $B$. brizantha $\mathrm{cv}$. Marandu, reconhecidas como as mais tolerantes à seca entre as espécies de Brachiaria, apresentaram taxas de alongamento das lâminas foliares bem inferiores às de $B$. mutica, sob a situação de déficit hídrico. A ocorrência de temperaturas abaixo do valor base mínimo, em torno de $10^{\circ} \mathrm{C}$ para espécies tropicais, segundo Almeida et al (1997), poderia ter causado efeito sinergístico entre temperatura e déficit hídrico, o que teria favorecido a $B$. mutica, em virtude de sua maior tolerância a baixas temperaturas.

Segundo Stür et al. (1996), a taxa de crescimento de espécies de Brachiaria começa a decrescer a partir de temperaturas médias abaixo de $24^{\circ} \mathrm{C}$ e a cessar à temperatura infra-ótima de $15^{\circ} \mathrm{C}$, em espécies menos tolerantes (B. humidicola e B. ruziziensis) e de $12^{\circ} \mathrm{C}$, em espécies tolerantes (B. decumbens e $B$. mutica).

Neste estudo, a temperatura do ar foi de 17,3 \pm $2,1^{\circ} \mathrm{C}$, bem abaixo de $24^{\circ} \mathrm{C}$, considerada baixa e comprometedora do crescimento das espécies de Brachiaria. Portanto, os baixos valores da temperatura mínima do ar $\left(11,3 \pm 3,2^{\circ} \mathrm{C}\right)$ reforçam a hipótese de que a temperatura também teria limitado o crescimento das espécies de Brachiaria, ainda que de forma distinta para cada espécie.

O potencial hídrico das lâminas foliares variou $(\mathrm{P}<0,05)$ com a situação de estresse apenas em 20/05 e 03/06 (Tabela 2).

Não se observou efeito dos estresses hídricos $(\mathrm{P}>0,05)$ sobre o potencial hídrico de lâminas foliares das espécies de Brachiaria (Tabela 2) nas avaliações realizadas em 20/05 (-0,06 MPa), 03/06 (-0,08 MPa), 07/06 (-0,07 MPa) e 14/06/1998 (-0,36 MPa); entretanto, em $11 / 06 / 98$, menor valor $(\mathrm{P}<0,05)$ foi observado em B. dictyoneura sob déficit hídrico (Tabela 3 ).

Salienta-se que o status hídrico das lâminas foliares das espécies de Brachiaria tendeu a decrescer de forma mais pronunciada a partir de 11/06/1998. Este comportamento parece ser mais acentuado sob déficit hídrico, principalmente em $B$. dictyoneura e B. mutica, sugerindo a ocorrência de algum mecanismo de ajuste em termos do status hídrico das lâminas foliares destas duas espécies.

A produção de matéria verde seca de lâminas foliares em B. brizantha acesso B-11 foi maior 
Tabela 2 - Potencial hídrico de lâminas foliares de espécies de Brachiaria, em diferentes datas de avaliação, em função da situação de estresse

Table 2 - Leaf blade water potential of the Brachiaria, in response to soil moisture condition on several dates

\begin{tabular}{lcccc}
\hline $\begin{array}{l}\text { Estresse } \\
\text { Stress }\end{array}$ & \multicolumn{5}{c}{$\begin{array}{c}\text { Data } \\
\text { Date }\end{array}$} \\
\cline { 2 - 5 } & $20 / 05$ & $03 / 06$ & $07 / 06$ & $14 / 06$ \\
\hline \multicolumn{5}{c}{$-\mathrm{Mpa}$} \\
DH & $0,07 \mathrm{a}$ & $0,07 \mathrm{a}$ & $0,07 \mathrm{a}$ & $0,41 \mathrm{a}$ \\
$\mathrm{AL}$ & $0,04 \mathrm{a}$ & $0,09 \mathrm{a}$ & $0,07 \mathrm{a}$ & $0,32 \mathrm{a}$ \\
\hline
\end{tabular}

${ }^{1}$ As abreviações são as mesmas da Tabela 1 (The symbols are the same as in Table 1).

$a>b$ : compara situações de estresse $(P<0,05)$ (compares types of stress $[P<.05])$.

Tabela 3 - Potencial hídrico de lâminas foliares das espécies de Brachiaria, em 11/06/1998, em função da natureza do estresse

Table 3 - Leaf blade water potential of the Brachiarias in response to soil water stress on 06/11/1998

\begin{tabular}{lcccccc}
\hline $\begin{array}{l}\text { Estresse } \\
\text { Stress }\end{array}$ & \multicolumn{7}{c}{$\begin{array}{c}\text { Espécie } \\
\text { Specie }\end{array}$} \\
\cline { 2 - 7 } & BD & BB & B-11 & BH & BI & BM \\
\hline \multicolumn{7}{c}{$-\mathrm{MPa}$} \\
\hline DH & $0,35 \mathrm{a}$ & $0,39 \mathrm{a}$ & $0,46 \mathrm{a}$ & $0,45 \mathrm{a}$ & $0,81 \mathrm{~b}$ & $0,65 \mathrm{a}$ \\
$\mathrm{AL}$ & $0,12 \mathrm{a}$ & $0,14 \mathrm{a}$ & $0,12 \mathrm{a}$ & $0,14 \mathrm{a}$ & $0,39 \mathrm{a}$ & $0,14 \mathrm{a}$
\end{tabular}

${ }^{1}$ As abreviações são as mesmas da Tabela 1 (The symbols are the same as in Table 1).

$a>b$ compara espécies $(P<0,05)$ (compares species $[p<.05])$.

$(\mathrm{P}<0,05)$ sob déficit hídrico que sob alagamento, ao contrário do que ocorreu em $B$. humidicola e $B$. dictyoneura, enquanto, em B. mutica, $B$. decumbens e $B$. brizantha $\mathrm{cv}$. Marandu, não houve variação com a situação de estresse (Tabela 4). Baruch (1994a) também não observou alteração nos valores da matéria seca de lâminas foliares em B. mutica com a variação da situação de estresse, ao contrário de outras gramíneas forrageiras tropicais.

As espécies de Brachiaria não diferiram entre si quanto à produção de matéria verde seca de lâminas foliares sob alagamento, mas sim sob déficit hídrico, de modo que a $B$. brizantha acesso B-11 superou a $B$. mutica, $B$. humidicola cv. Tupi e a $B$. dictyoneura, nesta característica. A maior sensibilidade ao alagamento, em termos de biomassa de lâminas foliares, em B. brizantha acesso B-11, decorre do acentuado efeito deste estresse sobre as taxas de alongamento e de senescência das lâminas foliares. Entretanto, este fato parece não se verificar de forma determinante em B. humidicola cv. Tupi e B. dictyoneura.

A produção de biomassa de lâminas foliares em B. humidicola $\mathrm{cv}$. Tupi poderia ter sido influenciada também pela taxa de aparecimento e alongamento antes da imposição dos tratamentos. No caso da B. dictyoneura, a produção de lâminas foliares poderia ser decorrente de variação da população de perfilhos.

A produção de matéria verde seca de colmos variou com a situação de estresse em $B$. brizantha acesso B-11, B. humdicola e B. mutica (Tabela 4). A $B$. mutica sobressaiu-se em termos de matéria verde seca de colmo, sobretudo em relação à $B$. brizantha cv. Marandu, que produziu pouca matéria seca de colmos, em ambas as situações de estresse. Baruch (1994a) também verificou maior produção de colmos sob alagamento que sob déficit hídrico. Portanto, podese inferir que a $B$. mutica é uma espécie que geralmente apresenta alta proporção de colmo na forragem, fenômeno que se intensifica sob alagamento.

A natureza do estresse afetou a produção de matéria verde seca da parte aérea apenas em $B$. brizantha acesso B-11, cuja parte aérea cresceu mais sob déficit hídrico, superando a $B$. humidicola cv. Tupi e B. dictyoneura (Tabela 4). A B. mutica, por sua vez, superou as demais espécies sob alagamento, em termos de matéria verde seca da parte aérea, à exceção da $B$. dictyoneura.

A superioridade da B. Brizantha, acesso B-11, quanto à produção de biomassa da parte aérea sob déficit hídrico, e da B. mutica sob alagamento decorre da maior participação de suas frações lâmina e colmo, respectivamente. Outro fato importante é que, tanto a $B$. mutica sob alagamento como a $B$. brizantha acesso B-11 sob déficit hídrico, se sobressaíram às demais espécies quanto a estas características, embora nem sempre com diferenças significativas. Portanto, ambos os fatos sugerem maior adaptação da $B$. brizantha acesso B-11 à seca e da B. mutica ao alagamento.

Haddade et al. (2002) também observaram superioridade da $B$. mutica sobre a $B$. decumbens para produção de biomassa sob alagamento, em condições de cultivo em vaso. Embora Comastri Filho (1994) tenha relatado superioridade da B. humidicola $\mathrm{cv}$. Tupi sobre a $B$. decumbens, quanto à matéria seca produzida, em solos suscetíveis à inundação no pantanal mato-grossense, não se verificou esta 
Tabela 4 - Biomassa da parte aérea, índice de área foliar, relação lâmina/colmo, altura de plantas e número de perfilhos aéreos e basilares, em função da situação de estresse

Table 4 - Shoot biomass, leaf area index, leaf/stem ratio, plant height, number of aerial and basal tillers, as a function of soil water status

\begin{tabular}{|c|c|c|c|c|c|c|}
\hline \multirow[t]{2}{*}{$\begin{array}{l}\text { Estresse }^{1} \\
\text { Stress }\end{array}$} & \multicolumn{6}{|c|}{$\begin{array}{l}\text { Espécie } \\
\text { Specie }\end{array}$} \\
\hline & $\mathrm{BD}$ & BB & B-11 & BH & BI & $\mathrm{BM}$ \\
\hline & \multicolumn{6}{|c|}{$\begin{array}{l}\text { Matéria verde seca de lâminas foliares }(\mathrm{kg} / \mathrm{ha}) \\
\text { Green leaf dry matter }(\mathrm{kg} / \mathrm{ha})\end{array}$} \\
\hline \multirow[t]{2}{*}{$\begin{array}{l}\mathrm{DH} \\
\mathrm{AL}\end{array}$} & $\begin{array}{l}1586 \mathrm{Aba} \\
1605 \mathrm{Aa}\end{array}$ & $\begin{array}{l}1705 \mathrm{ABa} \\
1216 \mathrm{Aa}\end{array}$ & $\begin{array}{l}2190 \mathrm{Aa} \\
1652 \mathrm{Ab}\end{array}$ & $\begin{array}{l}715 \mathrm{Cb} \\
1311 \mathrm{Aa}\end{array}$ & $\begin{array}{c}711 \mathrm{Cb} \\
1431 \mathrm{Aa}\end{array}$ & $\begin{array}{l}1139 \mathrm{BCa} \\
1154 \mathrm{Aa}\end{array}$ \\
\hline & \multicolumn{5}{|c|}{$\begin{array}{l}\text { Matéria verde seca de colmos }(\mathrm{kg} / \mathrm{hA}) \\
\text { Green stem dry matter }(\mathrm{kg} / \mathrm{ha})\end{array}$} & \\
\hline \multirow[t]{2}{*}{$\begin{array}{l}\mathrm{DH} \\
\mathrm{AL}\end{array}$} & $\begin{array}{l}1503 \mathrm{ABa} \\
1097 \mathrm{BCa}\end{array}$ & $\begin{array}{l}862 \mathrm{Ba} \\
669 \mathrm{Ca}\end{array}$ & $\begin{array}{l}1674 \mathrm{ABa} \\
872 \mathrm{BCb}\end{array}$ & $\begin{array}{l}1622 \mathrm{Aba} \\
1013 \mathrm{BCb}\end{array}$ & $\begin{array}{l}1336 \mathrm{Aba} \\
1568 \mathrm{Ba}\end{array}$ & $\begin{array}{l}2152 \mathrm{Ab} \\
3069 \mathrm{Aa}\end{array}$ \\
\hline & \multicolumn{5}{|c|}{$\begin{array}{l}\text { Matéria seca verde da parte aérea }(\mathrm{kg} / \mathrm{ha}) \\
\text { Green shoot dry matter }(\mathrm{kg} / \mathrm{ha})\end{array}$} & \\
\hline \multirow[t]{2}{*}{$\begin{array}{l}\mathrm{DH} \\
\mathrm{AL}\end{array}$} & $\begin{array}{l}3089 \mathrm{ABa} \\
2703 \mathrm{Ba}\end{array}$ & $\begin{array}{c}2567 \mathrm{ABa} \\
1886 \mathrm{Ba}\end{array}$ & $\begin{array}{l}3865 \mathrm{Aa} \\
2524 \mathrm{Bb}\end{array}$ & $\begin{array}{l}2336 \mathrm{Ba} \\
2324 \mathrm{Ba}\end{array}$ & $\begin{array}{l}2048 \mathrm{Ba} \\
2999 \mathrm{ABa}\end{array}$ & $\begin{array}{l}3291 \mathrm{ABa} \\
4223 \mathrm{Aa}\end{array}$ \\
\hline & \multicolumn{5}{|c|}{$\begin{array}{l}\text { Matéria morta seca da parte aérea }(\mathrm{kg} / \mathrm{ha}) \\
\text { Dead shoot biomass }(\mathrm{kg} / \mathrm{ha})\end{array}$} & \\
\hline \multirow[t]{2}{*}{$\begin{array}{l}\mathrm{DH} \\
\mathrm{AL}\end{array}$} & $\begin{array}{l}889 \mathrm{Ab} \\
2183 \mathrm{ABa}\end{array}$ & $\begin{array}{l}1223 \mathrm{Ab} \\
2335 \mathrm{Aa}\end{array}$ & $\begin{array}{c}789 \mathrm{Ab} \\
1944 \mathrm{ABCa}\end{array}$ & $\begin{array}{l}885 \mathrm{Aa} \\
1235 \mathrm{BCa}\end{array}$ & $\begin{array}{c}\text { 766Ab } \\
1843 \mathrm{ABCa}\end{array}$ & $\begin{array}{l}1133 \mathrm{Aa} \\
1216 \mathrm{Ca}\end{array}$ \\
\hline & \multicolumn{5}{|c|}{$\begin{array}{l}\text { Índice de área foliar } \\
\text { Leaf area index }\end{array}$} & \\
\hline \multirow[t]{2}{*}{$\begin{array}{l}\mathrm{DH} \\
\mathrm{AL}\end{array}$} & $\begin{array}{l}\text { 4,05Aba } \\
3,42 \mathrm{Aa}\end{array}$ & $\begin{array}{c}5,18 \mathrm{Aa} \\
2,50 \mathrm{ABb}\end{array}$ & $\begin{array}{c}5,19 \mathrm{Aa} \\
2,60 \mathrm{ABb}\end{array}$ & $\begin{array}{l}1,58 \mathrm{Ca} \\
1,69 \mathrm{Ba}\end{array}$ & $\begin{array}{c}1,52 \mathrm{Ca} \\
2,01 \mathrm{ABa}\end{array}$ & $\begin{array}{l}2,95 \mathrm{BCa} \\
3,11 \mathrm{ABa}\end{array}$ \\
\hline & \multicolumn{5}{|c|}{$\begin{array}{l}\text { Relação lâmina/colmo } \\
\text { Leaf/stem ratio }\end{array}$} & \\
\hline \multirow[t]{2}{*}{$\begin{array}{l}\mathrm{DH} \\
\mathrm{AL}\end{array}$} & $\begin{array}{l}1,03 \mathrm{Bb} \\
1,46 \mathrm{Ba}\end{array}$ & $\begin{array}{l}1,98 \mathrm{Aa} \\
1,88 \mathrm{Aa}\end{array}$ & $\begin{array}{l}1,31 \mathrm{Bb} \\
1,89 \mathrm{Aa}\end{array}$ & $\begin{array}{l}0,42 \mathrm{Cb} \\
1,31 \mathrm{Bca}\end{array}$ & $\begin{array}{c}0,48 \mathrm{Cb} \\
0,940 \mathrm{Ca}\end{array}$ & $\begin{array}{l}0,57 \mathrm{Ca} \\
0,39 \mathrm{Da}\end{array}$ \\
\hline & \multicolumn{5}{|c|}{$\begin{array}{l}\text { Altura de plantas }(\mathrm{m}) \\
\quad \text { Plant height }(\mathrm{m})\end{array}$} & \\
\hline \multirow[t]{2}{*}{$\begin{array}{l}\mathrm{DH} \\
\mathrm{AL}\end{array}$} & $\begin{array}{c}0,31 \mathrm{CDa} \\
0,27 \mathrm{Cb}\end{array}$ & $\begin{array}{l}0,33 \mathrm{Ca} \\
0,33 \mathrm{Ba}\end{array}$ & $\begin{array}{l}0,40 \mathrm{Ba} \\
0,36 \mathrm{Bb}\end{array}$ & $\begin{array}{l}0,28 \mathrm{Dea} \\
0,26 \mathrm{Ca}\end{array}$ & $\begin{array}{l}0,26 \mathrm{Ea} \\
0,23 \mathrm{Ca}\end{array}$ & $\begin{array}{l}0,50 \mathrm{Aa} \\
0,42 \mathrm{Ab}\end{array}$ \\
\hline & \multicolumn{5}{|c|}{$\begin{array}{l}\text { Número de perfilhos aéreos } / \mathrm{m}^{2} \\
\text { Aerial tiller number } / \mathrm{m}^{2}\end{array}$} & \\
\hline \multirow[t]{2}{*}{$\begin{array}{l}\mathrm{DH} \\
\mathrm{AL}\end{array}$} & $\begin{array}{l}469 \mathrm{Aa} \\
317 \mathrm{Ba}\end{array}$ & $\begin{array}{l}213 \mathrm{Aa} \\
240 \mathrm{Ba}\end{array}$ & $\begin{array}{l}395 \mathrm{Aa} \\
341 \mathrm{Ba}\end{array}$ & $\begin{array}{l}520 \mathrm{Aa} \\
413 \mathrm{Ba}\end{array}$ & $\begin{array}{l}475 \mathrm{Ab} \\
893 \mathrm{Aa}\end{array}$ & $\begin{array}{l}467 \mathrm{Ab} \\
891 \mathrm{Aa}\end{array}$ \\
\hline & \multicolumn{5}{|c|}{$\begin{array}{c}\text { Número de perfilhos basilares } / \mathrm{m}^{2} \\
\text { Basal tillers number } / \mathrm{m}^{2}\end{array}$} & \\
\hline $\begin{array}{l}\mathrm{DH} \\
\mathrm{AL}\end{array}$ & $\begin{array}{l}2333 \mathrm{Aa} \\
2555 \mathrm{Aa}\end{array}$ & $\begin{array}{l}1301 \mathrm{Ba} \\
1013 \mathrm{Ca}\end{array}$ & $\begin{array}{c}1267 \mathrm{Ba} \\
1333 \mathrm{BCa}\end{array}$ & $\begin{array}{c}2443 \mathrm{Aa} \\
2243 \mathrm{Aba}\end{array}$ & $\begin{array}{c}1923 \mathrm{ABb} \\
3117 \mathrm{Aa}\end{array}$ & $1117 \mathrm{Ba}$ \\
\hline
\end{tabular}

${ }^{1}$ As abreviações são as mesmas da Tabela 1 (The symbols are the same as in Table 1).

$\mathrm{A}>\mathrm{B}>\mathrm{C}>\mathrm{D}>\mathrm{E}$ compara espécies (compares species).

$a>b$ compara estresse $(P<0,05)$ (compares stress [ $p<.05])$.

superioridade da $B$. humidicola $\mathrm{cv}$. Tupi sobre a B. decumbens neste estudo.

A biomassa senescente da parte aérea sob alagamento foi maior $(\mathrm{P}<0,05)$ que sob déficit hídrico, exceto em B. humidicola cv. Tupi e B. mutica (Tabela 4). As espécies não diferiram quanto a este atributo, senão sob alagamento, condição em que $B$. mutica apresentou menores valores que 
B. decumbens e B. brizantha cv. Marandu.

Considerando que a altura do corte de uniformização foi a $10 \mathrm{~cm}$ acima do solo e o corte na colheita foi rente ao solo, acredita-se que a real quantidade de material vegetal morto foi superestimada. Portanto, a elevada quantidade de biomassa senescente da parte aérea explica-se, em parte, pelo "efeito residual", decorrente dos altos índices de área de lâminas foliares remanescentes ao corte de uniformização. As folhas de avançada idade, logo após o corte, caracterizaram-se pelo acentuado processo de senescência e morte, o que, evidentemente, se agravou durante o período de estresse, principalmente em $B$. brizantha $\mathrm{cv}$. Marandu e B-11 e B. decumbens sob alagamento.

O índice de área de lâminas foliares variou $(\mathrm{P}<0,05)$ com a natureza do estresse apenas em $B$. brizantha cv. Marandu e acesso B-11, que, sob alagamento, apresentaram área de lâminas foliares menor que sob déficit hídrico (Tabela 4).

Sob alagamento, a $B$. decumbens apresentou índice de área de lâmina foliar verde mais alto $(\mathrm{P}<0,05)$ que a $B$. mutica, além de tendência de superar as outras quatro espécies. Por outro lado, a $B$. brizantha cv. Marandu, B-11 e B. decumbens superaram a B. mutica, B. dictyoneura e B. humidicola cv. Tupi, sob déficit hídrico.

Baruch (1994a) também observou variação nas respostas ao tipo de estresse entre forrageiras tropicais. Cannell et al. (1985) também verificaram que o alagamento reduziu o índice de área de lâminas foliares em Avena sativa.

Maior número de perfilhos aéreos sob alagamento foi observado em $B$. mutica e $B$. dictyoneura (Tabela 4). Entretanto, quanto à produção perfilhos aéreos, as espécies não diferiram sob déficit hídrico.

Quanto ao número de perfilhos basilares, a $B$. dictyoneura sobressaiu-se sob algamento e a B. decumbens, sob déficit hídrico. No entanto, ambas se igualaram à $B$. humidicola cv. Tupi sob as duas situações de estresse (Tabela 4). Apesar disso, o acentuado perfilhamento em $B$. humidicola $\mathrm{cv}$. Tupi e $B$. dictyoneura não se traduziu em produção de perfilhos vigorosos, como verificado na $B$. decumbens.

A biomassa de sistema radicular somente foi avaliada sob déficit hídrico, situação em que a B. brizantha cv. Marandu e o acesso B-11 (médias de 3.060 e $3.173 \mathrm{~kg} / \mathrm{ha})$ se sobressaíram $(\mathrm{P}<0,05)$ à B. dictyoneura, com menor desenvolvimento de raízes (1.554 kg/ha), o que confirma a observação de campo,
Tabela 5 - Percentuais da biomassa aérea sob déficit hídrico e alagamento, relativamente à biomassa do corte de uniformização

Table 5 - Percentage figures of shoot biomass under low soil water potential and flooding relative to shoot biomass of uniformization cut

\begin{tabular}{lcccccc}
\hline $\begin{array}{l}\text { Estresse } \\
\text { Stress }\end{array}$ & \multicolumn{6}{c}{$\begin{array}{c}\text { Espécie } \\
\text { Specie }\end{array}$} \\
\cline { 2 - 7 } & BD & BB & B-11 & BH & BI & BM \\
\hline \multicolumn{7}{c}{$\%$ Biomassa } \\
& \multicolumn{7}{c}{ Biomass } \\
\hline $\mathrm{DH}$ & $25 \mathrm{BCa}$ & $13 \mathrm{Ca}$ & $37 \mathrm{Ba}$ & $36 \mathrm{Ba}$ & $75 \mathrm{Aa}$ & $21 \mathrm{BCa}$ \\
$\mathrm{AL}$ & $14 \mathrm{BCb}$ & $8 \mathrm{Ca}$ & $16 \mathrm{BCb}$ & $24 \mathrm{Ba}$ & $48 \mathrm{Aa}$ & $28 \mathrm{ABa}$ \\
\hline
\end{tabular}

${ }^{1}$ As abreviações são as mesmas da Tabela 1 (The symbols are the same as in Table 1).

$\mathrm{A}>\mathrm{B}>\mathrm{C}$ compara espécies (compares species).

$a>b$ compara estresse $(P<0,05)$ (compares stress $[p<.05])$.

acerca da maior tolerância à seca da $B$. brizantha $\mathrm{cv}$. Marandu.

Relativamente à produção obtida durante a fase de estabelecimento (Tabela 5), as espécies de Brachiaria sob alagamento produziram, percentualmente, menos matéria verde seca da parte aérea que sob déficit hídrico, refletindo o maior prejuízo causado pelo alagamento às forrageiras.

A $B$. dictyoneura apresentou a maior produção relativa de biomassa, inclusive quando comparada com a $B$. brizantha acesso B-11 e B. mutica, que apresentaram as maiores produções de matéria verde seca da parte aérea sob déficit hídrico e alagamento, respectivamente.

A seleção de espécies com alta produtividade no período das águas e menos sensível à falta e/ou ao excesso de água no solo seria bastante desejável para a produção de forragem. A B. dictyoneura apresentou baixa produção de biomassa, por ocasião da fase de estabelecimento, fato que explicaria sua alta produção relativa. Entretanto, esta aparente superioridade da $B$. dictyoneura quanto à produção relativa só seria vantajosa se sua produção de biomassa durante o período de estresse também fosse superior às outras espécies, fato que não ocorreu.

Jones et al. (1980) observaram que a produção de biomassa em $B$. brizantha, $B$. humidicola, $B$. mutica e $B$. decumbens, no período seco, representou apenas $43 \%$ da produção obtida no final do período chuvoso. 
Independentemente das espécies e dos acessos, as perdas de biomassa aérea por senescência e morte, sob condição de alagamento e déficit hídrico, alcançaram valores de 163,0 e $88,1 \%$, respectivamente, em relação às perdas observadas no corte de uniformização. Esta é outra evidência do maior efeito negativo do alagamento, relativamente ao déficit hídrico, sobre o acúmulo de forragem das Brachiarias. Todavia, as espécies mais afetadas em termos relativos foram a $B$. brizantha acesso B-11, B. humidicola cv. Tupi e $B$. dictyoneura, independentemente da situação do estresse. Mattos (2001) relatou acentuado comprometimento da taxa de alongamento e intensificação da taxa de senescência de lâminas foliares em quatro espécies de Brachiaria (B. decumbens, B. brizantha cv Marandu, B. humidicola cv. Tupi e B. mutica) cultivadas em vasos de PVC sob lâmina d'água de 10 $\mathrm{cm}$, além de comprometimento da fotossíntese líquida destas espécies, à exceção da B. mutica.

Os resultados observados neste trabalho reforçam o conhecimento corrente de que tanto a seca quanto o alagamento reduzem o potencial de produção de forragem, embora o alagamento comprometa mais a produção que o estresse hídrico.

\section{Conclusões}

O déficit hídrico e o alagamento não exerceram efeitos significativos sobre a maioria das características examinadas das espécies de Brachiaria. $\mathrm{O}$ alagamento foi mais prejudicial às características morfogênicas que o déficit hídrico, principalmente sobre a taxa de alongamento foliar. Os estresses hídricos exerceram efeitos diferenciados sobre as características produtivas das espécies estudadas. B. brizantha B-11 revelou-se a espécie mais adaptada às áreas sujeitas à seca, em virtude do seu grande sistema radicular e biomassa da parte aérea, decorrente, principalmente, da sua maior produção de lâminas foliares. A B. mutica revelou-se a melhor espécie para áreas sujeitas ao alagamento, em decorrência da maior altura de suas plantas e produção de biomassa, resultante, principalmente, de sua maior produção de colmos e raízes adventícias.

\section{Literatura Citada}

ALCÂNTARA, P.B. Origem das braquiárias e suas carterísticas morfológicas de interesse forrageiro. In: ENCONTRO SOBRE CAPINS DO GÊNERO BRACHIARIA, 1987, Nova Odessa. Anais... Nova Odessa: Instituto de Zootecnia, 1987. p.1-18.
ALMEIDA, E.X.; SETELICH, E.A; MARASCHIN, G.E. Oferta de forragem e variáveis morfogênicas em capim-elefante anão cv. Mott. In: REUNIÃO ANUAL DA SOCIEDADE BRASILEIRA DE ZOOTECNIA, 34., 1997, Juiz de Fora. Anais... Juiz de Fora: Sociedade Brasileira de Zootecnia, 1997. p.240-242.

ANDERSON, E.R. The reaction of seven Cenchrus ciliaris L. cultivars to flooding. Tropical Grasslands, v.8, n.1, p.33-39, 1974.

BANZATTO, D.A.; KRONKA, S.N. Experimentação agrícola. 7.ed. Jaboticabal: FUNEP, 1992. 249p.

BARUCH, Z. Responses to drought and flooding in tropical forage grasses. I. Biomass allocation, leaf growth and mineral nutrients. Plant and Soil, v.164, n.1, p.87-96, 1994a.

BARUCH, Z. Responses to drought and flooding in tropical forage grasses.II. Leaf water potential, photosynthesis rate and alcohol dehydrogenase activity. Plant and Soil, v.164, n.1, p.97-107, 1994b.

BEARD, J.B.; MARTIN, D.P. Influence of water temperature on submersion tolerance of four grasses. Agronomy Journal, v.62, n.2, p.257-259, 1970.

BITTMAN, S.; SIMPSON, G.M. Soil water deficit effect on yield, leaf area, and net assimilation rate of three forage grasses: crested wheatgrass, smooth bromegrass, and altai wildrye. Agronomy Journal, v.79, n.5, p.768-774, 1987.

CANNELL, R.Q.; BELFORD, R.K.; BLACKWELL, P.S. et al. Effects of waterlogging on soil aeration and on root and shoot growth and yield of winter oats (Avena sativa L.). Plant and Soil, v.85, n.3, p.361-373, 1985.

CHAPMAN, D.F.; LAMAIRE, G. Morphogenic and strutural determinants of plant regrowth after defoliation. In: INTERNATIONAL GRASSLAND CONGRESS, 17., 1993, New Zealand. Proceedings... New Zealand:[s. n.], 1993. p.95-104

COMASTRI FILHO, J.A. Avaliação de espécies de forrageiras nativas e exóticas na sub-região dos paiaguás no pantanal mato-grossense. Pesquisa Agropecuária Brasileira, v.29, n.6, p.971-978, 1994.

DAVIES, A. Tissue turnover in the sward. In: DAVIES, A. et al (Eds.) Sward measurement handbook. 2.ed. Hurley: BGS, 1993. p.183-216.

ETHERINGTON, J.R. Relationship between morphological adaptation to grazing, carbon balance and waterlogging tolerance in clones of Dactylis glomerata L. The New Phytologist, v.98, n.4, p.647-658, 1984.

HADDADE, I.R.; OBEID, J.A.; FONSECA, D.M. et al. Crescimento de espécies forrageiras tropicais submetidas a diferentes períodos de alagamento. Revista Brasileira de Zootecnia, v.31, n.5, p.1924-1930, 2002.

JONES, C.A.; PENA, D.; CARABALY, A. Effects of plant water potential, leaf diffusive resistance, rooting density and water use on the dry matter prodution of several tropical grasses during short periods of drought stress. Tropical Agriculture, v.57, n.3, 1980.

KLAR, A. E.; USBRTI JR., A.; HENDERSON, D. W. Differential responses of guinea grass populations to drought stress. Crop Science, v.18, n.5, p.853-857, 1978.

MATTOS, J.L.S. Avaliações morfológicas de espécies de Brachiaria sob diferentes disponibilidades de água no solo. Viçosa, MG: Universidade Federal de Viçosa, 2001. 122p. Tese (Doutorado em Zootecnia) - Universidade Federal de Viçosa, 2001. 
McIVOR, J.G. Leaf growth and senescence in Urochloa mosambicensis and U. oligotricha in a seasonally dry tropical environment. Australian Journal Agriculture Research, v.35, p.177-187, 1984.

SOARES FILHO, C.V. Recomendações de espécies e variedades de Brachiaria para diferentes condições. In: SIMPÓSIO SOBRE MANEJO DE PASTAGEM, 11., 1994, Piracicaba. Anais... Piracicaba: Fundação de Estudos Agrários Luiz de Queiroz, 1994. p.25-48.

STUR, W.W.; HOPKINSON, J.M.; CHEN, C.P. Regional experience whith Brachiaria: Asia, the south pacific, and Australia. In: MILES et al. (Eds) Brachiaria: biology, agronomy, and improvement. Cali: CIAT, 1996. p.258-271.

VALLE, C.B.; EUCLIDES, V.P.B.; MACEDO, M.C.M Características das plantas forrageiras do gênero Brachiaria. In: SIMPÓSIO SOBRE MANEJO DE PASTAGEM, 17., 2000, Piracicaba. Anais... Piracicaba: Fundação de Estudos Agrários Luiz de Queiroz, 2000. p.65-108.
WATSON, E.R.; LAPINS, P.; BARRON, R.J.W. Effect of waterlogging on the growth, grain and straw yield of wheat, barley, and oats. Australian Journal Experimental Agriculture Animal Husbandry, v.16, p.114-122, 1976.

WEIGAND, R.; Stamato Neto, J.; COELHO, R.D. Pasto irrigado produz mais. ANUALPEC, 1998. p.45-50.

ZIMMER, A.H.; EUCLIDES, V.P.B. Importância das pastagens para o futuro da pecuária de corte no Brasil. In: SIMPÓSIO DE FORRAGICULTURA E PASTAGEM, 1., 2000, Lavras. Anais... Lavras: Universidade Federal de Lavras, 2000. p.1-49.

Recebido em: 16/08/04

Aceito em: $23 / 03 / 05$ 